\title{
Dosimetric Comparison and Clinical Toxicity in Cervical Cancer Patients Treated with Intensity- Modulated and Three-Dimensional Conformal Radiotherapy: Real-World Data
}

\author{
Emmanuel Kwateng Drokow ${ }^{1,2}, \mathrm{Zi} \mathrm{Liu}^{2, *}$, Tao Wang ${ }^{2}$, Jin $\mathrm{Su}^{2}$, Wei Yuan ${ }^{2}$, Fan Shi ${ }^{2}, \mathrm{Yi} \mathrm{Li}^{2}$, Evans Sasu ${ }^{4}$, Lanlan $\mathrm{Xu}^{1}$ and Gloria \\ Selorm Akpabla ${ }^{3}$ \\ 'Department of Radiation Oncology, Zhengzhou University People's Hospital \& Henan Provincial People's Hospital Henan, Zhengzhou, PR China \\ ${ }^{2}$ Department of Radiation Oncology, First Affiliated Hospital of Xi'an Jiaotong University, Xi'an-Shaanxi, China \\ ${ }^{3}$ Department of Internal Medicine, Tianjin Medical University, Tianjin - China \\ ${ }^{4}$ National Centre for Radiotherapy \& Nuclear Medicine, Korle Bu Teaching Hospital, Accra, Ghana
}

${ }^{\star}$ Corresponding author: Prof Liu Zi, M.D., Ph.D., Department of Radiation Oncology, First Affiliated Hospital of Xian Jiaotong University,277\# West Yanta Road, Xi’an, Shaanxi, China; Email: liuzimail@163.com

Received: October 16, 2020; Accepted: October 29, 2020; Published: November 18, 2020

\begin{abstract}
Background: Predominantly used in external beam radiotherapy (EBRT) are intensity modulated radiotherapy (IMRT) and three-dimensional conformal radiotherapy (3DCRT). However, the superiority between these two techniques remains inconclusive. This study aim to evaluate the late clinical toxicity of cervical cancer patients treated with intensity-modulated radiation therapy (IMRT) compared to three-dimensional conformal radiation therapy (3D-CRT) and dosimetrically compare the planning target volume (PTV) plan of 3D-CRT to the PTV plan of IMRT based on target coverage and bladder and rectum doses at different volumes.

Methods and Materials: From September 2011 - December 2015, 146 patients with International Federation of Gynaecology and Obstetrics (FIGO) stage IB2-IIB squamous cell carcinoma of the cervix were analysed retrospectively. The patients received EBRT of 50 Gy in 25 fractions of the whole pelvic delivered with IMRT or 3D-CRT. Seventy-five (75) patients received 3D-CRT and seventy-one (71) patients received IMRT.

Results: The 2 years' overall survival (OS) was $92 \%$ in the IMRT group and $88 \%$ in the 3D-CRT group (p-value $=0.073$ ). The disease-free survival outcome (DFS) was $83 \%$ and $80 \%$ in IMRT and 3D-CRT group respectively (p-value). Acute genitourinary (GU) and gastrointestinal (GI) toxicity were lower in IMRT patients compared to 3D-CRT patients (GU, $21.1 \%$ vs. $37.3 \%$, p-value $=0.179 ; \mathrm{GI}, 46.5 \%$ vs. $49.3 \%$, p-value $=0.436$ ). The mean coverage of the prescribed dose in IMRT and 3D-CRT techniques was $50.02 \pm 0.10 \mathrm{~Gy}$ and $50.10 \pm 0.23 \mathrm{~Gy}$ respectively with a non-significant p-value of 0.005 for 95 percent $\left(D_{95}\right)$ of the PTV. Also, the mean coverage of $5 \%\left(D_{5}\right)$ of the PTV was $52.66 \pm 0.39$ Gy and $53.89 \pm 0.76$ Gy of the prescribed dose in IMRT and 3D-CRT techniques respectively with a significant p-value of 0.0001 .
\end{abstract}

Conclusion: Patients treated with IMRT had a lower dose to bladder and rectum, a lesser rate of late toxicity and comparable clinical outcome than 3D-CRT. We admonish larger sample size studies and longer follow-up in subsequent studies to affirm our results.

Keywords: Cervical cancer; IMRT; 3D-CRT; Rectum; Bladder

\section{Introduction}

Cancer has become a significant public health problem in China since 2010 due to increasing incidence and mortality, making it the number one cause of death in the country [1,2]. External beam radiotherapy (EBRT) is a vital method of treatment for cervical cancer management. Most often than not EBRT and brachytherapy in addition to chemotherapy are often used when treating and managing locally advanced cancer of the cervix. The primary goal of EBRT is in the delivering of maximum radiation to the malignant tissue, with minimum radiation to healthy organs. This treatment can be noxious, and about $20-25 \%$ of patients are reported to have severe side effects [3]. Hence, reliable dose-response knowledge in malignant lesions and organs at risk (OAR) is therefore very vital. Before advancement into new treatment planning and imaging technique, most cervical cancer patients were treated using 2D (Two-dimensional) planning. In $2 \mathrm{D}$ treatment planning, the contour of the patient is captured with $\mathrm{x}$-ray using lead wire, and bony landmarks and is transcribed on a graph paper sheet with an identified reference point, [4] which results in the target volume being inadequately uncovered. With the 
limitation of 2D planning, 3D treatment planning and conformal radiotherapy became the standard for EBRT in the 90ths [5]. This treatment planning uses computer tomography (CT) scan images with patients required to be positioned in the planning set-up and requires a computerised treatment planning system (TPS). 3D-CRT is a form of EBRT which uses computers and unique imaging technologies to optimize the radiation beams precisely in other to reduce radiation to surrounding healthy tissues; and was started to be used for effective management of patients since it could give a maximum target coverage and also has the tendency for dose optimization to normal healthy tissues. It makes use of several high photon beams to amply deliver a high dose to a centrally located target volume with minimum dose to superficial structures in the pelvis. Intensity-modulated radiation therapy (IMRT) allows radiation to be more precisely shaped to fit the target volume by using heterogeneous fluences beams from different directions thereby optimises high radiation dose to the target volume and also limiting the amount of radiation received by the normal healthy organs. With IMRT, the beam intensity is able to be optimised as it orients around the patients using computer algorithms [6]. The 'inverse method' in treatment planning forms the basis of this process hence able to generate significant dose gradients in the adjacent structures and target volume to accomplish dose-volume prescription [7]. In IMRT, many beams with varying intensity levels are used in treating the tumour while 3D-CRT uses uniform intensity radiation beams hence the constraint of the latter is evident whenever a tumour is wrapped around an organ. Many experts indicated that IMRT is capable of reducing doses to the bone marrow, rectum and bowel and are linked with reduced levels of haematological, gastrointestinal (GI) and genitourinary (GU) toxicity compared to conventional radiation therapy. Nevertheless, these studies were usually defined by small sample sizes and the absence of clinical outcome data. Additionally, brachytherapy patients were involved in their selection criteria and this could influence toxicity. Retrospective reviews comparing IMRT and 3D-CRT technique for cervical cancer patients treated by radiotherapy are deficient, and also there has been inconsistency finding in dose to OAR. The purpose of this study was to analyse retrospectively the clinical toxicity of cervical cancer patients treated with IMRT compared to 3D-CRT and secondly, to compare the PTV plans of 3D-CRT to the PTV plan of IMRT on the basis of target coverage and doses to bladder and rectum at different volumes.

\section{Materials and Methods}

\section{Patient Selection}

146 stage IB2-stage IIB cervical cancer patients were treated from September 2011-December 2015. The eligibility criteria were [8]:

i. Biopsy confirmation of squamous cell carcinoma or adenocarcinoma.

ii. Cytological /histological diagnosis of cervical cancer.

iii. No previous surgery, chemotherapy or radiation.

iv. No evidence of distance metastasis.

v. KPS performance score 70-80.

\section{Pre-Treatment Evaluation}

The pre-treatment workup included a comprehensive medical history, vagina-recto-abdominal examination. Radiological studies like CT-scan of the abdomen-pelvis, chest $\mathrm{x}$-ray and MRI in a few selected patients. Laboratory studies included a complete blood count (CBC), Liver function test (LFT), Blood Chemistries, BUN/ $\mathrm{Cr}$, SCC blood test. The clinical-stage was defined according to the International Federation of Obstetrics \& Gynaecology (FIGO) staging system.

\section{CT-Simulation}

All patients were immobilised with a thermoplastic sheet and underwent CT simulation for planning in a supine position. Philips CT scanner was used for simulation and $3 \mathrm{~mm}$ slice images of the abdomen and pelvis area were obtained. The Pinnacle treatment planning system (TPS) (Version 9.2) was used for planning and target contouring.

\section{Treatment Planning}

The clinical target volume (CTV) and organs at risk (OAR) were contoured using the concept and definition of volume targets from ICRU reports $[9,10]$. The gross tumour volume (GTV) and clinical target volume (CTV) were contoured on each single axial CT slice. The CTV included palpable tumour and areas expected to be affected with subclinical tumours. Therefore, the CTV included the pelvic lymph node (external, internal and common iliac), cervix, vagina upper section and uterus. A margin of $10 \mathrm{~mm}$ was generated around the CTV to define the planning target volume (PTV). Four fields (two lateral and PA-AP fields) with zero-degree $\left(0^{\circ}\right)$ couch angle were used to generate the $3 \mathrm{D}$-CRT plans (Figure 1). The isocenter was positioned at the PTV's geometric centre. 10 megavolt (MV) photon energy was used for all plans to improve coverage of PTV and reduce dose to the skin. The beam aperture was shaped to the PTV in each beam's eye view and a margin of $0.5 \mathrm{~cm}$ in all directions accounting for the beam penumbra. The PTV was prescribed a total dose of 50 Gy (2Gy per fraction). The bladder and rectum were protected with a 4 -cm central shield after 40 Gy. IMRT plans were generated using 10 megavolt energy with six coplanar fields (Figure 2). Patients had whole pelvic radiotherapy prescribe to $50 \mathrm{~Gy}$ with either 3D-CRT or IMRT in 1.8-2 Gy per fractions from Monday - Friday. Chemotherapy involving cisplatin $\left(25 \mathrm{mg} / \mathrm{m}^{2}\right)$ was given concurrently to all patients from second to fifth week during radiotherapy treatment. None of the patients received high dose rate-intracavitary brachytherapy.

\section{Plan Evaluation}

All plans were passed and accepted after more than $95 \%$ of the PTV received more than $95 \%$ of the dose prescribed (PD). The dose-volume histograms (DVHs) were used in evaluating the PTV coverage, rectum and bladder between 3D-CRT and IMRT plans. The parameter analysed for bladder and rectum included $\mathrm{D}_{15} \mathrm{D}_{50} \mathrm{D}_{80}$ (dose to $15 \%, 50 \%$ and $80 \%$ of organ volume) while PTV coverage was based on $\mathrm{D}_{5}$ and $\mathrm{D}_{95}$ (Dose to $5 \%$ and $95 \%$ of the PTV respectively). The conformity index (CI) and homogeneity index (HI) was calculated in both techniques using the formulae below. 
Prof Kai Sun (2020) Dosimetric Comparison and Clinical Toxicity in Cervical Cancer Patients Treated with Intensity-Modulated and ThreeDimensional Conformal Radiotherapy: Real-World Data

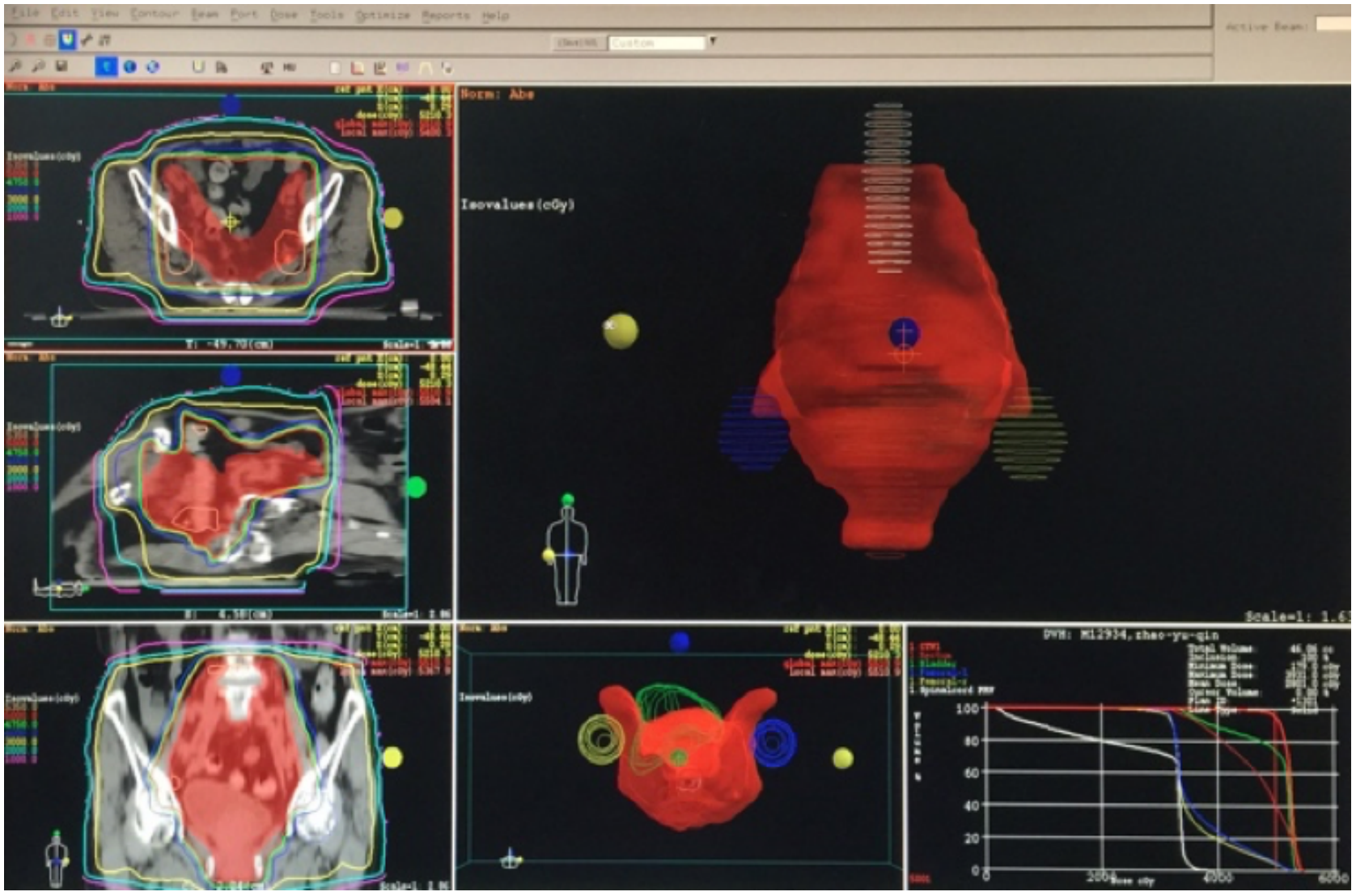

Figure 1: Shows the 4-field beam arrangement and isodose curve in 3D-CRT.

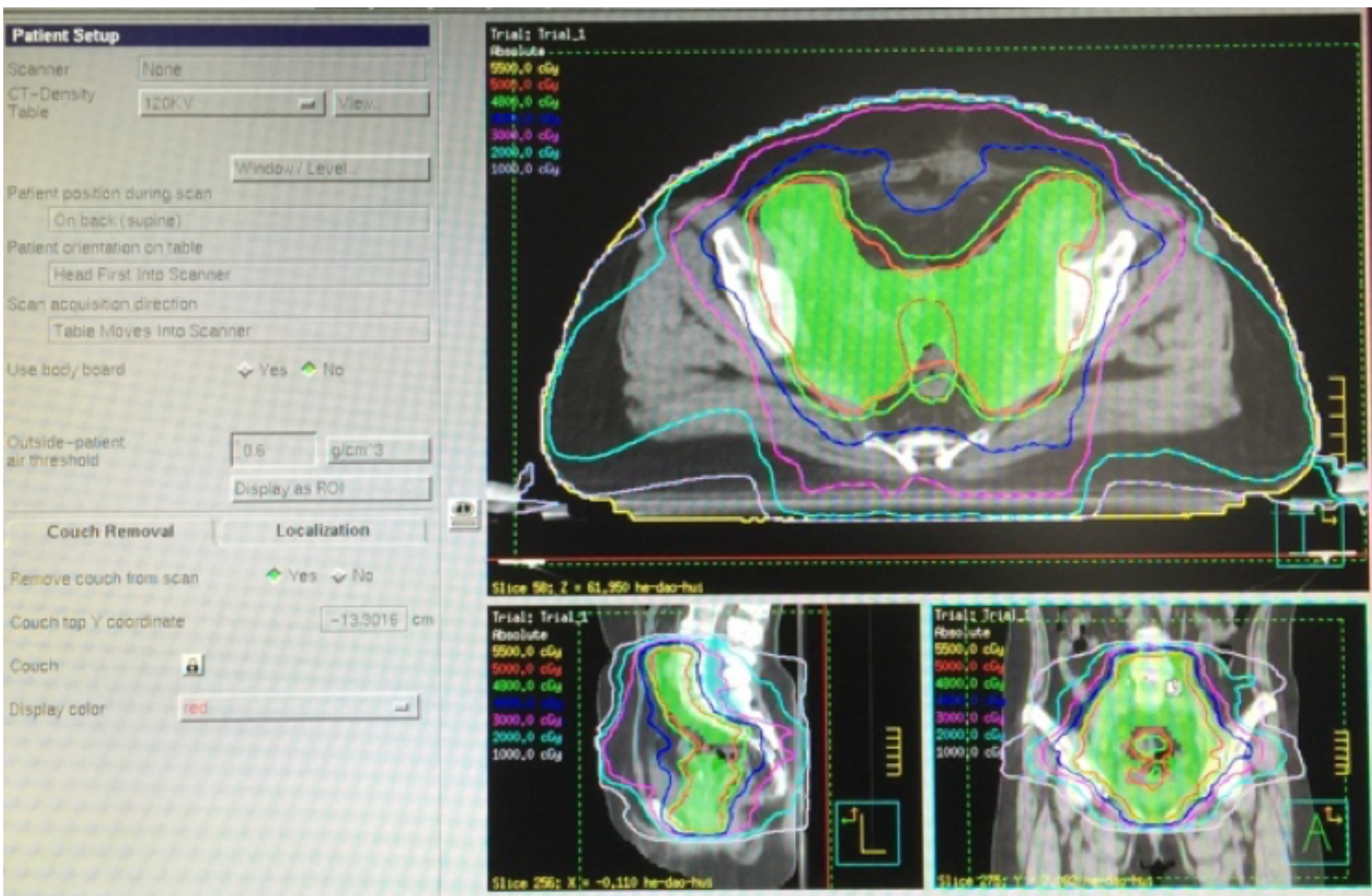

Figure 2: Shows the beam arrangement and isodose curve in IMRT technique. 
Prof Kai Sun (2020) Dosimetric Comparison and Clinical Toxicity in Cervical Cancer Patients Treated with Intensity-Modulated and Three-

Dimensional Conformal Radiotherapy: Real-World Data

$\mathrm{HI}_{95 \%}=\mathrm{D}_{5} / \mathrm{D}_{95}$; where $\mathrm{D}_{5}$ is the minimum dose of $5 \%$ of the target volume indicating the maximum dose, and $\mathrm{D}_{95}$ is the maximum dose of $95 \%$ of the target volume indicating the minimum dose. The Homogeneity Index (HI) is an accurate method for analysing the homogeneity of the target volume dose distribution. HI, therefore, demonstrates in all terminology the ratio between both the minimum and maximum dose in the target volume and the lower value demonstrates a more homogeneous distribution of the dose within this volume.

The ideal value is 1 , and it increases as the plan become less homogeneous.

$\mathrm{CI}_{95 \%}=$ Total volume receiving $95 \%$ of prescribed dose/planning target volume. The ideal value is 1 .

\section{Statistical Analysis}

All statistical analyses were carried out using SPSS 18, and a substantial difference in each set of dosimetric variables was determined using an independent sample test and chi-square. The rate of survival was evaluated after treatment was completed. The Kaplan Meier method was used to calculate overall survival (OS) and diseasefree survival (DFS). With the aid of the log-rank test, the significance of the difference was analyzed and a p-value $<0.05$ was considered significant statistically.

\section{Follow-Up}

One month after treatment, patients had a gynaecological examination and pelvic CT/MRI. Afterwards, they were followed at a regular interval of 3 months for the first 2 years and at an interval of 6 months thereafter and then once a year. Version 3.0 of the Common Terminology Criteria for Adverse Events (CTCAE) was used in evaluating chronic and acute toxicity.

\section{Results}

\section{Characteristics and Treatment of patients}

146 stage IB2-stage IIB cervical cancer patients were treated from September 2011-December 2015. Seventy-five (75) were treated with $3 \mathrm{D}-\mathrm{CRT}$ and the median age was 50 years (range, 39-68). Seventy-one (71) were also treated with IMRT and the median age was 53 years (range, 32-78). The squamous cell carcinoma histology type was seen in one hundred and thirty-seven $(137,93.8 \%)$ patients and nine (9, $6.2 \%)$ patients with adenocarcinoma. Table 1 shows a summary of the patients' characteristics.

\section{Dose-Volume Histogram (DVH) Outcomes}

The $95 \%$ PTV mean value was $50.02 \pm 0.10 \mathrm{~Gy}$ and $50.10 \pm 0.23 \mathrm{~Gy}$ of the prescribed dose in IMRT and 3D-CRT techniques respectively with a significant p-value of 0.005 . Also, the mean coverage of $5 \%$ of the PTV was $52.66 \pm 0.34 \mathrm{~Gy}$ and $53.89 \pm 0.76 \mathrm{~Gy}$ of the prescribed dose in IMRT and 3D-CRT techniques respectively with a significant $\mathrm{p}$-value of 0.001 . Hence the target coverage was esteemed satisfactory and appropriate in both groups.

The HI mean value was $1.052 \pm 0.008$ and $1.083 \pm 0.021$ in IMRT and $3 \mathrm{D}$-CRT plans respectively, and the p-value 0.001 , indicates the statistical significance of $\mathrm{HI}$ in both plans. The $\mathrm{CI}$ mean value was 1.330 \pm 0.103 and $1.109 \pm 0.214$ in IMRT and 3D-CRT plans respectively, with a significant $0.001 \mathrm{p}$-value. Table 2 shows the outcomes of the CI, $\mathrm{HI}$ and target coverage in both treatment technique.

The dose received by $15 \%\left(\mathrm{D}_{15}\right), 50 \%\left(\mathrm{D}_{50}\right)$ and $80 \%\left(\mathrm{D}_{80}\right)$ of the bladder in IMRT was $51.30 \mathrm{~Gy}, 46.79 \mathrm{~Gy}$ and $38.69 \mathrm{~Gy}$ respectively while that of 3D-CRT was also $52.96 \mathrm{~Gy}, 51.30 \mathrm{~Gy}$ and $41.95 \mathrm{~Gy}$ at $\mathrm{D}_{15}, \mathrm{D}_{50}$ and $\mathrm{D}_{80}$ respectively. The dose difference between these two techniques at $\mathrm{D}_{15}, \mathrm{D}_{50}$ and $\mathrm{D}_{80}$ was highly significant with $\mathrm{p}$-value 0.0001 at all level. Furthermore, dose received by $15 \%\left(D_{15}\right), 50 \%\left(D_{50}\right)$ and $80 \%\left(\mathrm{D}_{80}\right)$ of the rectum in IMRT was $51.04 \mathrm{~Gy}, 48.82 \mathrm{~Gy}$ and 43.72 Gy respectively while that of 3D-CRT was also $52.24 \mathrm{~Gy}, 50.99$ Gy and $48.08 \mathrm{~Gy}$ at $\mathrm{D}_{15}, \mathrm{D}_{50}$ and $\mathrm{D}_{80}$ respectively. The dose difference between these two techniques at $\mathrm{D}_{15}, \mathrm{D}_{50}$ and $\mathrm{D}_{80}$ was highly significant with p-value 0.001 at all level. Table 3 shows the detailed values of rectum and bladder dose at $\mathrm{D}_{15}, \mathrm{D}_{50}$ and $\mathrm{D}_{80}$.

Table 1: Patients clinical characteristics.

\begin{tabular}{|c|c|c|c|}
\hline Characteristics & IMRT & 3D-CRT & p-value \\
\hline \multicolumn{4}{|l|}{ Age } \\
\hline Median & 53 & 50 & \\
\hline Range & $32-78$ & $39-68$ & \\
\hline \multicolumn{4}{|l|}{ Histology type } \\
\hline SCC & $64(93.8 \%)$ & $73(97.3 \%)$ & \\
\hline Adenocarcinoma & $7(9.9 \%)$ & $2(2.7 \%)$ & 0.071 \\
\hline \multicolumn{4}{|l|}{ Stage } \\
\hline IB2 & $6(8.5 \%)$ & $7(9.3 \%)$ & \\
\hline IIA1 & $29(40.8 \%)$ & 44 (58.7\%) & \\
\hline IIA2 & $2(2.8 \%)$ & $2(2.7 \%)$ & 0.131 \\
\hline IIB & $34(47.9 \%)$ & $22(29.3 \%)$ & \\
\hline \multicolumn{4}{|l|}{ Grade } \\
\hline 1 & $8(11.3 \%)$ & $12(16.0 \%)$ & 0.166 \\
\hline 2 & $59(83.1 \%)$ & $53(70.7 \%)$ & \\
\hline 3 & $5(5.6 \%)$ & $10(13.3 \%)$ & \\
\hline \multicolumn{4}{|l|}{ Tumour Size } \\
\hline$<4 \mathrm{~cm}$ & $45(63.4 \%)$ & $50(66.7 \%)$ & 0.677 \\
\hline$\geq 4 \mathrm{~cm}$ & $26(36.6 \%)$ & $25(33.3 \%)$ & \\
\hline \multicolumn{4}{|l|}{ LVSI } \\
\hline Yes & $30(42.3 \%)$ & $39(52.0 \%)$ & 0.238 \\
\hline No & $41(57.7 \%)$ & $36(48.0 \%)$ & \\
\hline \multicolumn{4}{|l|}{ Pelvic Node } \\
\hline Yes & $19(26.8 \%)$ & $17(22.7 \%)$ & 0.540 \\
\hline No & $52(73.2 \%)$ & $57(76.0 \%)$ & \\
\hline
\end{tabular}

Table 2: Outcomes of the CI, HI and target coverage in both treatment technique.

\begin{tabular}{|l|c|c|c|}
\hline Dosimetric Parameters & IMRT & 3D-CRT & P-value \\
\hline $\mathrm{D}_{5}$ & $52.66 \pm 0.39$ & $53.89 \pm 0.76$ & 0.001 \\
\hline $\mathrm{D}_{95}$ & $50.02 \pm 0.10$ & $50.10 \pm 0.23$ & 0.005 \\
\hline $\mathrm{CI}$ & $1.330 \pm 0.103$ & $1.109 \pm 0.214$ & 0.001 \\
\hline $\mathrm{HI}$ & $1.052 \pm 0.008$ & $1.083 \pm 0.021$ & 0.001 \\
\hline
\end{tabular}


Prof Kai Sun (2020) Dosimetric Comparison and Clinical Toxicity in Cervical Cancer Patients Treated with Intensity-Modulated and Three-

Dimensional Conformal Radiotherapy: Real-World Data

\section{Survival Outcome and Failure Patterns}

The 2 years' overall survival (OS) was $92 \%$ in the IMRT group and $88 \%$ in the 3D-CRT group with a non-significate p-value of 0.073 and the median follow-up time was 28 months. The disease-free survival outcome (DFS) was $83 \%$ and $80 \%$ in IMRT and 3D-CRT group respectively. Locoregional failure was noticed in 5 patients. Three (3) from the 3D-CRT group and 2 from the IMRT group. Distant metastasis was observed in one patient in the three-dimensional conformal radiotherapy group in addition to the locoregional failure. Six (6) death rate was recorded during the follow-up, two (2) from the IMRT group and 4 from the 3D-CRT group. The causes of death were pulmonary embolism ( 1 patient), heart failure (3 patients) and natural death (2).

\section{Clinical Toxicity Outcome}

Table 4 shows the percentage of patients with acute genitourinary (GU), haematological and gastrointestinal (GI) toxicity and their grades. Less acute genitourinary (GU) and gastrointestinal (GI) toxicity were noticed in the IMRT patients compared to the 3D-CRT patients ( $p$-value $=0.436$ and 0.179 respectively). None of the patients experienced grade 4 genitourinary (GU) and gastrointestinal (GI) toxicity in both groups. Two patients in the IMRT category developed

Table 3: Summary of rectum and bladder dose.

\begin{tabular}{|l|c|c|c|}
\hline Dosimetric Parameters & IMRT & 3D-CRT & P-value \\
\hline Bladder & & & \\
\hline$D_{15}$ & 51.300 .39 & $52.96 \pm 0.88$ & 0.001 \\
\hline$D_{50}$ & $46.79 \pm 2.28$ & $51.30 \pm 1.72$ & 0.001 \\
\hline$D_{80}$ & $38.69 \pm 3.63$ & $41.95 \pm 6.14$ & 0.001 \\
\hline Rectum & & & \\
\hline$D_{15}$ & $51.04 \pm 0.52$ & $52.24 \pm 0.89$ & 0.001 \\
\hline$D_{50}$ & $48.82 \pm 0.97$ & $50.99 \pm 0.75$ & 0.001 \\
\hline$D_{80}$ & $43.72 \pm 2.59$ & $48.08 \pm 2.97$ & 0.001 \\
\hline
\end{tabular}

Table 4: Clinical toxicity between IMRT and 3D-CRT.

\begin{tabular}{|l|c|c|c|c|c|}
\hline Toxicity & Grade & 3D-CRT arm, n (\%) & IMRT arm, n (\%) & $\mathbf{x}^{2}$ & p-value \\
\hline Hematologic & 0 & $43(57.3 \%)$ & $47(5.3 \%)$ & 1.834 & 0.608 \\
\hline & 1 & $21(28.0 \%)$ & $18(25.4 \%)$ & & \\
\hline & 2 & $8(10.7 \%)$ & $4(5.6 \%)$ & & \\
\hline GI & 3 & $3(4.0 \%)$ & $2(2.8 \%)$ & & \\
\hline & 0 & $47(62.7 \%)$ & $56(78.9 \%)$ & 4.907 & 0.179 \\
\hline & 1 & $22(29.3 \%)$ & $12(16.9 \%)$ & & \\
\hline & 2 & $5(6.7 \%)$ & $2(2.8 \%)$ & & \\
\hline GU & 3 & $1(1.3 \%)$ & $1(1.4 \%)$ & & \\
\hline & 0 & $28(37.3 \%)$ & $22(31.0 \%)$ & 2.726 & 0.436 \\
\hline & 1 & $33(44.0 \%)$ & $31(43.7 \%)$ & & \\
\hline & 2 & $10(13.3 \%)$ & $16(22.5 \%)$ & & \\
\hline & 3 & $4(5.3 \%)$ & $2(2.8 \%)$ & & \\
\hline Edema & Yes & $12(16.0 \%)$ & $2(2.8 \%)$ & 7.311 & 0.007 \\
\hline & No & $63(84.0 \%)$ & $69(97.2 \%)$ & & \\
\hline
\end{tabular}

oedema while 12 patients in the 3D-CRT category experienced the same effect. None significant statistical difference was noticed between the two groups when the various clinical toxicity was considered.

\section{Discussion}

Previous epidemiological studies have shown that most cervical cancer patients mostly report to the hospital in advance stages of the disease. The public, accepted management for locally advanced cervical cancer (LACC) is brachytherapy with concurrent cisplatin chemoradiotherapy. Conventional radiotherapy continues to be the golden standard for LACC. There has been a reduction in the clinical outcomes and toxicities of IMRT compared with 3D-CRT from preliminary studies. The utilisation of IMRT for gynaecologic tumours including locally advanced cervical cancer has upsurge over these years even though there is insufficient retrospective randomised data to support its usage. From our results, both techniques attained the desired target coverage since $95 \%$ of the PTV had above $95 \%$ of the prescribed dose (PD). Also, there was better CI, HI and PTV coverage in IMRT compared to 3D-CRT because IMRT uses computer optimised intensity beams and multiple beam angles. Secondly, by using computer algorithms, the intensity of the beam can be optimised in IMRT as it orients around the patient, therefore, allowing radiation to be more precisely shaped to fit the target volume. The results of previous studies, when compared to this present study, confirmed that both IMRT and 3D-CRT are useful in PTV coverage hence no difference in our PTV coverage when compared with previous studies. Van De Bunt et al. [11] reported that IMRT is superior to conformal and conventional treatment in sparing critical organs with ample target volume coverage and also stated that IMRT remains superior after EBRT of $30 \mathrm{~Gy}$ regardless of internal organ movement and tumour deterioration.

Mell et al. [12], reported IMRT that there was a reduction in doses to the bone marrow and small bowel when patients were treated with IMRT. A study by Naik et al. [13], reported that doses to organ volume of bladder and rectum were reduced in IMRT patients compared to 3D-CRT. Fiorino et al. [14] concluded that IMRT was superior regarding bowel sparing for doses above 30Gy and also a correlation exists between toxicity and the amount of radiation received by an organ. Central target volume boost is possible with IMRT for patients whom brachytherapy is not possible due to a reduction in doses to OAR thereby allowing higher dose up to 66-70 Gy to be delivered using IMRT. Retrospective studies have accounted that decrease in dose to healthy organs may present a clinical benefit in clinical toxicities reduction. Jereczek - Fossa et al. [15] examined 317 postoperative endometrium carcinoma patients and reported that there was a statistically significant correlation between late and acute bowel reactions. The morbidity and complications among cervical cancer patients after a long-term treatment survivor was assessed by Kamal et al. [16] and reported that the rate of obstruction of the small intestines was comparable in IMRT and 3D-CRT with no significant p-value in both groups. Ajeet et al. [17] reported grade 2 diarrhoea, tenesmus and constipation in patients treated with 3D-CRT compared to a lower grade in IMRT patients. Avinash et al. [18] concluded that there were no differences in both techniques when 
Prof Kai Sun (2020) Dosimetric Comparison and Clinical Toxicity in Cervical Cancer Patients Treated with Intensity-Modulated and Three-

Dimensional Conformal Radiotherapy: Real-World Data

the grade of haematological toxicities was considered every week even though there was a statistically significant difference between IMRT and 3D-CRT during the second week when the total count and Neutrophils count were assessed. Our results showed that less acute genitourinary (GU) and gastrointestinal (GI) toxicity was noticed in the IMRT patients compared to the 3D-CRT patients (p-value $=0.436$ and 0.179 respectively). None of the patients experienced grade 4 genitourinary (GU) and gastrointestinal (GI) toxicity in both groups. Two patients in the IMRT category developed oedema while 12 patients in the 3D-CRT category experienced the same effect. In general, lower clinical toxicities were observed in the IMRT patients than the 3D-CRT patients even though there wasn't any statistical significance between the two techniques.

Past studies [19-27] in postoperative patients treated with IMRT have normally shown suitable survival outcomes. Chen et al. [28] analyzed 35 patients receiving four-field radiation therapy and 33 patients receiving intensity-modulated radiotherapy and concluded that IMRT improved locoregional control. An update of the study of the Gynaecologic Oncology Group showed 3-year overall survival and progression-free survival rates of $88 \%$ and $86 \%$ respectively in stage IB cervical cancer patients. Results from the Radiation Therapy Oncology Group 0418 study, involving 48 patients showed an estimated 2-year OS and DFS rates of $94.6 \%$ and $86.9 \%$ respectively with a median follow-up duration of 2.68 years. In Folkert et al. [29] studies involving 34 patients, the 3 years OS was $91.1 \%$ and the 5 years DFS was $91.2 \%$. Our findings were similar to this study.

Our study's major limitation is the short follow-up period. Furthermore, using bone marrow-sparing methods could reduce the higher rates of haematological toxicity recorded in treated patients with intensity-modulated radiotherapy. In addition, more focus should be given to the target margin in order to leave an adequate margin in IMRT planning for PTV expansion.

\section{Conclusion}

In conclusion, patients treated with IMRT had a lower dose of bladder and rectum, a lesser rate of clinical toxicity and comparable clinical outcome than 3D-CRT. We admonish larger sample size studies and longer follow-up in subsequent studies to affirm our results.

\section{References}

1. Shan J (2014) Cervical cancer increases among young Chinese women.

2. Ostor AG, Rome RM (1994) Micro-invasive squamous cell carcinoma of the cervix: a clinico-pathologic study of 200 cases with long-term follow up. Int J Gynecol Cancer 4: 257-264.

3. Chen W, Zheng R, Baade PD, Zhang S, Zeng H, et al. (2016) Cancer statistics in China, 2015. CA Cancer J Clin 66: 115-132.

4. Hochman A, Ratzkowski E, Schrieber H (1955) Incidence of carcinoma of cervix in Jewish women in Israel. Br J Cancer 9: 358-364.

5. Perez CA (1998) Uteri cervix. In: Perez CA, Brady LW, Principles and practice of radiation oncology 3rd edition. Philadelphia, PA: Lippincott-Raven Publishers 1998: 1733-1834.

6. Low D, Purdy JA, Perez CA et al. (1992) Intensity modulated radiation therapy, In: Levitt SH, Potish RA, Khan FM, Perez CA eds. Levitt and Tapley's technological basis of radiation therapy: Clinical application 3rd edition. Baltimore, MD: Lippincott Williams and Wilkins 1992: 126-146.
7. Khan FM (2007) Treatment Planning in Radiation Oncology, 2nd edition, Philadelphia: Lippincott Williams and Wilkins.

8. Drokow EK, Zi L, Qian H, Xu L, Foli F, et al. (2020) Tolerability, efficacy and feasibility of concurrent gemcitabine and cisplatin (CGP) combined with intensity modulated radiotherapy for loco-regionally advanced carcinoma of the cervix. Journal of Cancer 11: 2632-2638. [crossref]

9. ICRU Report 50 (1993) Prescribing, recording and reporting photon beam therapy: ICRU, Bethesda, MD,

10. ICRU Report 62 (1999) Prescribing, recording and reporting photon beam therapy (Supplement to ICRU report 50): ICRU, Bethesda, MD.

11. Van de Bunt L, van der Heide UA, Ketelaars M, de Kort GA, Jurgenliemk-Schulz IM (2006) Conventional, conformal, and intensity-modulated radiation therapy treatment planning of external beam radiotherapy for cervical cancer: the impact of tumor regression. Int J Radiat Oncol Biol Phys 64: 189-196. [crossref]

12. Mell LK, Tiryaki H, Ahn KH, John C Roeske, Bulent Aydogan et al. (2008) Dosimetric comparison of bone marrow-sparing intensity-modulated radiotherapy versus conventional techniques for treatment of cervical cancer. Int J Radiat Oncol Biol Phys 71: 1504-1510. [crossref]

13. A Naik, O.P. Gurjar, P. Bagdare et al. (2016) Dosimetric comparison between IMRT and 3D-CRT planning. Int. J. Radiat 14: 189-196.

14. Fiorino C, Alongi F, Perna L Et al. (2009) Dose-volume relation- ships for acute bowel toxicity in patients treated with pelvic nodal irradiation for prostate cancer. Int J Radiat Oncol Biol Phys 75: 29-35. [crossref]

15. Jereczek-Fossa BA, Jassem J, Badzio A (2002) Relationship between acute and late normal tissue injury after postop- erative radiotherapy in endometrial cancer. Int $J$ Radiat Oncol Biol Phys 52: 476-482. [crossref]

16. Kamal A Elghamrawi (2011) Treatment complications among long-term survivors of cervical cancer: treated by surgery or radiotherapy. Oncology Reviews 5: 261-266.

17. Gandhi AK, Sharma DN, Rath GK, Julka PK, Subramani V, et al. (2013) Early clinical outcomes and toxicity of intensity modulated versus conventional pelvic radiation therapy for locally advanced cervix carcinoma: a prospective randomized study. Int $J$ Radiat Oncol Biol Phys 87: 542-548. [crossref]

18. Avinash HU, Arul Ponni TR, Janaki M G, Kirthi Koushik A S, Kumar SM (2015) A prospective dosimetric and clinical comparison of acute haematological toxicities in intensity modulated radiation therapy and three-dimensional conformal radiotherapy with concurrent chemotherapy in carcinoma cervix. J Can Res Ther 11: 83-87. [crossref]

19. Hui Beina MD, Zhang, Yingbing MD, Shi Fan MD, Wang Juan PhD, et al. (2014) Association Between Bone Marrow Dosimetric Parameters and Acute Hematologic Toxicity in Cervical Cancer Patients Undergoing Concurrent Chemoradiotherapy: Comparison of Three-Dimensional Conformal Radiotherapy and IntensityModulated Radiation Therapy. International Journal of Gynecological Cancer 24: 1648-1652. [crossref]

20. Wang YM, Wang CJ, Fang FM, Chen HC, Hsu HC, et al. (2017) Differences in the outcomes and complications between elderly and younger uterine cervical cancer patients treated by definitive radiotherapy - a propensity score-matched study. Gynecol Oncol 145:277-283.

21. Hui B, Zhang Y, Shi F, Wang J, Wang T, et al. (2014) Association between bone marrow dosimetric parameters and acute hematologic toxicity in cervical cancer patients undergoing concurrent chemoradiotherapy: comparison of three-dimensional conformal radiotherapy and intensity modulated radiation therapy. Int J Gynecol Cancer 24:1648-1652. [crossref]

22. Chang Y, Yang ZY, Li GL, Li Q, Yang Q, et al. (2016) Correlations between radiation dose in bone marrow and haematological toxicity in patients with cervical cancer: a comparison of 3DCRT, IMRT, and Rapid ARC. Int J Gynecol Cancer 26:770-776. [crossref]

23. Mell LK, Sirak I, Wei L, Tarnawski R, Mahantshetty U, et al. (2017) Bone marrowsparing intensity modulated radiation therapy with concurrent cisplatin for stage IB-IVA cervical cancer: an international multicenter phase II clinical trial (INTERTECC-2). Int J Radiat Oncol Biol Phys 97:536-545. [crossref]

24. Tanderup K, Fokdal LU, Sturdza A, Haie-Meder C, Mazeron R, et al. (2016) Effect of tumor dose, volume and overall treatment time on local control after radiochemotherapy including MRI guided brachytherapy of locally advanced cervical cancer. Radiother Oncol 120:441-446. [crossref] 
Prof Kai Sun (2020) Dosimetric Comparison and Clinical Toxicity in Cervical Cancer Patients Treated with Intensity-Modulated and ThreeDimensional Conformal Radiotherapy: Real-World Data

25. Sturdza A, Potter R, Fokdal LU, Haie-Meder C, Tan LT, et al. (2016) Image guided brachytherapy in locally advanced cervical cancer: improved pelvic control and survival in RetroEMBRACE, a multi-center cohort study. Radiother Oncol 120: 428433. [crossref]

26. Weiping W, Xiaorong H, Junfang Y, Jie S, et al. (2017) Outcome and toxicity of radical radiotherapy or concurrent chemoradiotherapy for elderly cervical cancer women. BMC Cancer 17:510. [crossref]

27. Mei-Ling LAN, Xian YU, He XIAO et al. (2016) Clinical outcomes and toxicity of postoperative intensity-modulated versus three-dimensional conformal radiation therapy in patients with cervical cancer. Asia-Pacific Journal of Clinical Oncology 12: 430-436. [crossref]
28. Chen MF, Tseng CJ, Tseng CC, Yu CY, Wu CT, et al. (2008) Adjuvant concurrent chemoradiotherapy with intensity modulated pelvic radiotherapy after surgery for high-risk, early stage cervical cancer patients. Cancer J 14: 200-206. [crossref]

29. Folkert MR, Shih KK, Abu-Rustum NR et al. (2013) Postoperative pelvic intensitymodulated radiotherapy and concurrent chemotherapy in intermediate- and highrisk cervical cancer. Gynecol Oncol 128: 288-293. [crossref]

\section{Citation:}

Emmanuel Kwateng Drokow, Zi Liu, Tao Wang, Jin Su, Wei Yuan, et al. (2020) Dosimetric Comparison and Clinical Toxicity in Cervical Cancer Patients Treated with Intensity-Modulated and Three-Dimensional Conformal Radiotherapy: Real-World Data. Cancer Stud Ther J Volume 5(3): 1-7. 